\title{
ON GROWTH RATE OF COEFFICIENTS IN BERNSTEIN POLYNOMIALS FOR THE STANDARD MODULUS FUNCTION ON A SYMMETRIC INTERVAL
}

\author{
M.A. PETROSOVA, I.V. TIKHONOV, V.B. SHERSTYUKOV
}

\begin{abstract}
The subject of the paper is closely related to one general direction in the approximation theory, within which the growth rate of the coefficients of algebraic polynomials is studied for uniform approximations of continuous functions. The classical Bernstein polynomials play an important role here. We study in detail a model example of Bernstein polynomials for the standard modulus function on a symmetric interval. The question under consideration is the growth rate of of the coefficients in these polynomials with an explicit algebraic representation. It turns out that in the first fifteen polynomials the growth of the coefficients is almost not observed. For the next polynomials the situation changes, and coefficients of exponential growth appear. Our main attention is focused on the behaviour of the maximal coefficient, for which exact exponential asymptotics and corresponding two-sided estimates are established (see Theorem 2). As it follows from the obtained result, the maximal coefficient has growth $2^{n / 2} / n^{2}$, where $n$ is the index of the Bernstein polynomial. It is shown that the coefficients equidistant from the maximal one have a similar growth rate (for details, see Theorem 3). The group of the largest coefficients is located in the central part of the Bernstein polynomials but at the ends the coefficients are sufficiently small. The behavior of the sum of absolute values of all coefficients is also considered. This sum admits an explicit expression that is not computable in the sense of traditional combinatorial identities. On the base of a preliminary recurrence relation, we succeeded to obtain the exact asymptotics for the sum of absolute values of all coefficients and to give the corresponding two-sided estimates (see Theorem 4). The growth rate of the sum is $2^{n / 2} / n^{3 / 2}$. In the end of the paper, we compare this result with a general Roulier estimate and new related problems are formulated.
\end{abstract}

Keywords: standard modulus function, Bernstein polynomials, growth of coefficients.

Mathematics Subject Classification: 41A10, 11B83, 05A10

\section{INTRODUCTION}

There is one special direction in the approximation theory devoted to studying possible growth rate of the coefficients in algebraic polynomials used in uniform approximations of continuous functions on a given set [1]-[7]. Some results, see [3]-[6], were obtained by estimating the coefficients in the corresponding Bernstein polynomials. As a rule, such estimates were given independent of specific approximated functions and this is why they had quite "rought" character. A choice of a specific example allows one to specify essentially the real growth rate of the coefficients in Bernstein polynomials.

M.A. Petrosova, I.V. Tikhonov, V.B. Sherstyukov, On growth rate of Coefficients in BernSTEIN POLYNOMIALS FOR THE STANDARD MODULUS FUNCTION ON A SYMMETRIC INTERVAL.

(c) Petrosova M.A., Tikhonov I.V., Sherstyukov V.B. 2018.

The reported study was partially funded by RFBR according to the research project 18-01-00236.

Submitted December 17, 2017. 
For instance, in work [8], there was found an explicit algebraic expression for Bernstein polynomials in the power of a variable $x$ for the function $f(x)=|2 x-1|$ considered on the segment $[0,1]$. It was shown that the maximal coefficient in this expression grows exponentially approximately as $2^{3 n / 2}$, where $n$ is the index of a Bernstein polynomial. A series of additional facts on the coefficients of Bernstein polynomials on the standard segment $[0,1]$ was mentioned in work [9].

It is curious that while extending the Bernstein construction on the symmetric segment $[-1,1]$, a nature of such results changes. Features of a symmetric segment in the theory of Bernstein polynomials were found out rather recently, see [10], [11. In particular, in work [11] there was found an explicit algebraic expression in powers of the variable $x$ for Bernstein polynomials for the standard modulus function $f(x)=|x|$ on the symmetric segment $[-1,1]$. A final formula turned out to be simple and elegant. Here we employ the results of [11] to estimate the growth rate in rising polynomials.

For general information on the theory of Bernstein polynomials we refer to textbooks [12][16].

\section{Formulation of PROBlem}

Given a function $f \in C[-1,1]$, the Bernstein polynomials on the symmetric segment $[-1,1]$ are introduced by the rule:

$$
B_{n}(f, x)=\frac{1}{2^{n}} \sum_{k=0}^{n} f\left(\frac{2 k}{n}-1\right) C_{n}^{k}(1+x)^{k}(1-x)^{n-k}, \quad n \in \mathbb{N} .
$$

Here $C_{n}^{k}$ are binomial coefficients. Choosing the standard modulus function

$$
f(x)=|x|, \quad x \in[-1,1]
$$

formula (1) becomes

$$
B_{n}(x)=\frac{1}{2^{n}} \sum_{k=0}^{n}\left|\frac{2 k}{n}-1\right| C_{n}^{k}(1+x)^{k}(1-x)^{n-k}, \quad n \in \mathbb{N} .
$$

Polynomials (3) are denoted simply by $B_{n}(x)$ without mentioning the function $f$ since in the present work we consider no other generating functions except (2).

Inter alia, polynomials (3) obey a special gluing rule meaning

$$
B_{2 m+1}(x)=B_{2 m}(x), \quad m \in \mathbb{N} .
$$

Identity (4) is a reflection of the general law of pairwise coincidence, which is true for Bernstein polynomials on $[-1,1]$ for piecewise linear functions with rational abscissas of breakpoints, see [10], [11]. Due to (4) we consider only polynomials with even indices:

$$
B_{2 m}(x)=\frac{1}{2^{2 m}} \sum_{k=0}^{2 m}\left|\frac{k}{m}-1\right| C_{2 m}^{k}(1+x)^{k}(1-x)^{2 m-k}, \quad m \in \mathbb{N} .
$$

Let us proceed to the algebraic writing in powers of the variable $x$. 
Opening brackets and collecting like terms, for the first polynomials we obtain

$$
\begin{gathered}
B_{2}(x)=\frac{1}{2}\left(1+x^{2}\right), \\
B_{4}(x)=\frac{1}{8}\left(3+6 x^{2}-x^{4}\right), \\
B_{6}(x)=\frac{1}{16}\left(5+15 x^{2}-5 x^{4}+x^{6}\right), \\
B_{8}(x)=\frac{1}{128}\left(35+140 x^{2}-70 x^{4}+28 x^{6}-5 x^{8}\right), \\
B_{10}(x)=\frac{1}{256}\left(63+315 x^{2}-210 x^{4}+126 x^{6}-45 x^{8}+7 x^{10}\right), \\
B_{12}(x)=\frac{1}{1024}\left(231+1386 x^{2}-1155 x^{4}+924 x^{6}-495 x^{8}+154 x^{10}-21 x^{12}\right)
\end{gathered}
$$

and so forth. All polynomials $B_{2 m}(x)$ for even function (2) involve only even powers of $x$, see [10], and have the sturcture

$$
B_{2 m}(x)=\sum_{k=0}^{m} a_{2 m, 2 k} x^{2 k}, \quad m \in \mathbb{N} .
$$

Explicit expression showing exact form of formula (6) is as follows:

$$
B_{2 m}(x)=2^{-2 m} C_{2 m}^{m}\left[1+\sum_{k=1}^{m} \frac{(-1)^{k-1}}{2 k-1} C_{m}^{k} x^{2 k}\right], \quad m \in \mathbb{N} .
$$

Representation (7) was found in work [11. We are going to employ (7) for studying the coefficients of the polynomials $B_{2 m}(x)$.

We briefly call the parameter $n=2 m$ index. Comparing (6) and (7), we obtain

$$
\begin{aligned}
a_{2 m, 0}=2^{-2 m} C_{2 m}^{m}, & m \in \mathbb{N}, \\
a_{2 m, 2 k}=2^{-2 m} C_{2 m}^{m}(-1)^{k-1} \beta_{m}(k), \quad m & \in \mathbb{N}, \quad k=1, \ldots, m,
\end{aligned}
$$

with positive numbers

$$
\beta_{m}(k)=\frac{1}{2 k-1} C_{m}^{k}, \quad m \in \mathbb{N}, \quad k=1, \ldots, m .
$$

The behavior of free coefficients (8) is given by the known asymptotics

$$
2^{-2 m} C_{2 m}^{m} \sim \frac{1}{\sqrt{\pi m}}, \quad m \rightarrow \infty,
$$

meaning a "slow" vanishing as $m \rightarrow \infty$.

We focus our attention on main coefficients (9). Given a fixed $n=2 m$, we want to find a maximal (by the absolute value) coefficient among (9) and to estimate it behavior as the index $n=2 m$ increases.

We introduce the main characteristics:

$$
\mu_{2 m} \equiv \max _{1 \leqslant k \leqslant m}\left|a_{2 m, 2 k}\right|=2^{-2 m} C_{2 m}^{m} \max _{1 \leqslant k \leqslant m} \beta_{m}(k), \quad m \in \mathbb{N},
$$

with the numbers $\beta_{m}(k)$ in formula $(10)$. Let us study the behavior of quantity (12).

For the first polynomials mentioned above we obviously have

$$
\mu_{2}=\frac{1}{2}, \quad \mu_{4}=\frac{3}{4}, \quad \mu_{6}=\frac{15}{16}, \quad \mu_{8}=\frac{35}{32}, \quad \mu_{10}=\frac{315}{256}, \quad \mu_{12}=\frac{693}{512},
$$

and all these values are attained at the coefficient at $x^{2}$. 
The next polynomial

$$
B_{14}(x)=\frac{1}{2048}\left(429+3003 x^{2}-3003 x^{4}+3003 x^{6}-2145 x^{8}+1001 x^{10}-273 x^{12}+33 x^{14}\right)
$$

is special since the value

$$
\mu_{14}=\frac{3003}{2048}
$$

is attained at three coefficients simultaneously: at $x^{2}, x^{4}$ and $x^{6}$.

It could seem that the quantity $\mu_{2 m}$ does not exhibit a tendency to a special growth since all values (13), (14) are located in a narrow range from $1 / 2$ to $3 / 2$. But the real picture becomes more complicated: the main "strategic" tendency in the behavior of $\mu_{2 m}$ becomes evident only for sufficiently large incides $n=2 \mathrm{~m}$. It is quite problematic to find out such tendency basing on an initial definition (5) and not using explicit writing (7). This is a principal difference of the situation with the function $f(x)=|x|$ on $[-1,1]$ from a similar example $f(x)=|2 x-1|$ on $[0,1]$, where a swift growth of the coefficients is evidently seen while opening the brackets already at the first indices of Bernstein polynomials (see [8]). We proceed to precise formulations.

\section{MAIN STATEMENTS}

We employ notations (9), (10), 12) for quantities $a_{2 m, 2 k}, \beta_{m}(k), \mu_{2 m}$, respectively. In order to estimate $\mu_{2 m}$, for each fixed $m \in \mathbb{N}$ we need to the find the maximal of numbers $\beta_{m}(k)$. Let us find out the laws acting in a finite sequence $\beta_{m}(k)$ with a fixed $m \geqslant 2$ as $k=1,2, \ldots, m$.

The initial case $m=1$ is not of interest since the sequence obviously degenerates into the only element $\beta_{1}(1)=1$.

Theorem 1. Let the quantity $\beta_{m}(k)$ be defined by formula (10). Subject to $m \geqslant 2$, one should distinguish three cases.

1. For each fixed $m \in\{2,3,4,5,6\}$, the numbers $\beta_{m}(k)$ form a finite strictly decreasing sequence with the maximal element $\beta_{m}(1)=m$.

2. As $m=7$, the numbers $\beta_{7}(k)$ form a special set

$$
7, \quad 7, \quad 7, \quad 5, \quad \frac{7}{3}, \frac{7}{11}, \frac{1}{13},
$$

where the first three elements coincide and then the elements strictly decay.

3. For each fixed $m \geqslant 8$ a finite sequence $\beta_{m}(k)$ strictly increases to $k=[(m-1) / 2]$ and then it strictly decreases, that is, the maximal element of the sequence is exaclty $\beta_{m}([(m-1) / 2])$.

The mentioned features allows us to explain why the first Bernstein polynomials (7) corresponding to the values $m$ from 1 to 7 are exceptional and growth of coefficients $(9)$ is almost not observed. But as $m \geqslant 8$ the tendency changes. The main result is as follows.

Theorem 2. Let the quantity $\mu_{2 m}$ be defined by formula (12) as the maximal absolute value of the coefficients (9) taken from polynomials (7). Then the asymptotic formula holds:

$$
\mu_{2 m} \sim \frac{\sqrt{2}}{\pi} \frac{2^{m}}{m^{2}}, \quad m \rightarrow \infty
$$

and the estimate

$$
\frac{\sqrt{2}}{\pi} \frac{2^{m}}{m^{2}}<\mu_{2 m}<1.2215 \cdot \frac{\sqrt{2}}{\pi} \frac{2^{m}}{m^{2}}
$$

is true for all natural $m \geqslant 8$. 
Making estimate (17) a little bit rougher, we can write a more demonstrable version:

$$
0.45 \frac{2^{m}}{m^{2}}<\mu_{2 m}<0.55 \frac{2^{m}}{m^{2}}, \quad m \in \mathbb{N}, \quad m \geqslant 8
$$

Relations (16)-(18) show that the maximal (by the absolute value) coefficient in polynomials (7) grows with at most exponential rate but this rate is essentially small than in the analogous example on the standard segment $[0,1]$ (cf. [8], [9], [15]). We proceed to the proof of the formulated results.

\section{Proof OF ThEOREM 1}

For a fixed natural $m \geqslant 2$ we consider the numbers

$$
\beta_{m}(1), \quad \beta_{m}(2), \quad \ldots, \quad \beta_{m}(m),
$$

taken from formula $(10)$. We need to characterise the growth and decay of the elements in finite sequence 19 . In order to do this, we define the relations:

$$
q_{m}(k)=\frac{\beta_{m}(k+1)}{\beta_{m}(k)}, \quad k=1, \ldots, m-1 .
$$

We need to compare numbers 20 with one. We write in an expanded form:

$$
q_{m}(k)=\frac{C_{m}^{k+1}}{2 k+1} \cdot \frac{2 k-1}{C_{m}^{k}}=\frac{(2 k-1)(m-k)}{(2 k+1)(k+1)}=\frac{-2 k^{2}+(2 m+1) k-m}{2 k^{2}+3 k+1}
$$

and we consider the differences

$$
q_{m}(k)-1=\frac{-2 k^{2}+(2 m+1) k-m}{2 k^{2}+3 k+1}-1=-\frac{4 k^{2}-2(m-1) k+m+1}{2 k^{2}+3 k+1} .
$$

We introduce an auxiliary quadratic function

$$
h_{m}(t)=4 t^{2}-2(m-1) t+m+1, \quad t \in \mathbb{R} .
$$

The above formula imply obviously the following result.

Lemma 1. For each $k \in\{1, \ldots, m-1\}$, the relation $q_{m}(k)<1$ is equivalent to the relation $h_{m}(k)>0$; the relation $q_{m}(k)>1$ is equivalent to the relation $h_{m}(k)<0$; and finally, the relation $q_{m}(k)=1$ is equivalent to the identity $h_{m}(k)=0$.

To apply Lemma 1, we study quadratic function (21); by $D_{m}$ we denote its discriminant. Since $D_{m} / 4=m^{2}-6 m-3$, then $D_{m}<0$ as

$$
m \in\{2,3,4,5,6\}
$$

and $D_{m}>0$ for other $m \geqslant 7$. First we consider $m$ in set (22). Since for such $m$ the values of function (21) are strictly positive, then $h_{m}(k)>0$ for all $k=1, \ldots, m-1$. Hence, by Lemma 1, we conclude that

$$
q_{m}(k)=\frac{\beta_{m}(k+1)}{\beta_{m}(k)}<1, \quad k=1, \ldots, m-1 .
$$

Therefore, for each $m$ in 22 , sequence of numbers 19 strictly decreases and

$$
\max _{1 \leqslant k \leqslant m} \beta_{m}(k)=\beta_{m}(1)=C_{m}^{1}=m, \quad m \in\{2,3,4,5,6\} .
$$

This proves first statement of Theorem 1.

The case $m=7$ is special. Function (21) becomes

$$
h_{7}(t)=4 t^{2}-12 t+8=4(t-1)(t-2) .
$$


It is clear that $h_{7}(1)=h_{7}(2)=0$ and $h_{7}(k)>0$ as $k=3,4,5,6$. Respectively,

$$
q_{7}(1)=\frac{\beta_{7}(2)}{\beta_{7}(1)}=1, \quad q_{7}(2)=\frac{\beta_{7}(3)}{\beta_{7}(2)}=1, \quad \text { but then } \quad q_{7}(k)=\frac{\beta_{7}(k+1)}{\beta_{7}(k)}<1
$$

as $k=3,4,5,6$. As a result we get sequence 19 with three maximal elements:

$$
\beta_{7}(1)=\beta_{7}(2)=\beta_{7}(3)>\beta_{7}(4)>\beta_{7}(5)>\beta_{7}(6)>\beta_{7}(7) .
$$

Exact values are given in formula (15). This proves second statement of Theorem 1.

We proceed to the main case $m \geqslant 8$. Here $D_{m} / 4=m^{2}-6 m-3>0$ and function (21) possesses two different roots:

$$
t_{1} \equiv t_{1, m}=\frac{m-1-\sqrt{m^{2}-6 m-3}}{4}, \quad t_{2} \equiv t_{2, m}=\frac{m-1+\sqrt{m^{2}-6 m-3}}{4},
$$

at that, $t_{1}<t_{2}$ and $t_{1}+t_{2}=(m-1) / 2$. We observe that

$$
\left\{\begin{array}{l}
h_{m}\left(\frac{1}{2}\right)=1-(m-1)+m+1=3>0, \\
h_{m}(1)=4-2(m-1)+m+1=7-m<0 .
\end{array}\right.
$$

Therefore, the root $t_{1}$ satisfies the bounds $\frac{1}{2}<t_{1}<1$. But then

$$
\frac{m-3}{2}<t_{2}=\frac{m-1}{2}-t_{1}<\frac{m-2}{2} .
$$

We define an integer quantity:

$$
j_{m} \equiv\left[\frac{m-1}{2}\right] .
$$

Employing estimate (23), we are going to establish the following fact.

Lemma 2. For each natural $m \geqslant 8$ the relation holds:

$$
j_{m}-1<t_{2} \equiv t_{2, m}=\frac{m-1+\sqrt{m^{2}-6 m-3}}{4}<j_{m},
$$

that is, the root $t_{2}$ is localized in the interval $\left(j_{m}-1, j_{m}\right)$ with $j_{m}$ given by (24).

Proof. We employ an elementary inequality

$$
\frac{m-2}{2} \leqslant j_{m} \equiv\left[\frac{m-1}{2}\right] \leqslant \frac{m-1}{2},
$$

which holds for each natural (and even integer) $m$. This yields:

$$
j_{m}-1 \leqslant \frac{m-1}{2}-1=\frac{m-3}{2}<\frac{m-2}{2} \leqslant j_{m} .
$$

Comparing with estimate (23) being valid for all natural $m \geqslant 8$, we arrive at (25). The proof is complete.

We also observe that for each $m \geqslant 8$, quantity $j_{m}$ is located in the segment

$$
3 \leqslant j_{m} \equiv\left[\frac{m-1}{2}\right] \leqslant m-5 .
$$

Taking into consideration localization 25) of root $t_{2}$ and the fact that $\frac{1}{2}<t_{1}<1$, we obtain the values:

$$
\begin{aligned}
& h_{m}(1)<0, \quad h_{m}(2)<0, \quad \ldots, \quad h_{m}\left(j_{m}-1\right)<0, \\
& h_{m}\left(j_{m}\right)>0, \quad h_{m}\left(j_{m}+1\right)>0, \ldots, \quad h_{m}(m-1)>0 .
\end{aligned}
$$


Then, employing Lemma 1, we infer that

$$
q_{m}(k)=\frac{\beta_{m}(k+1)}{\beta_{m}(k)}>1, \quad k=1, \ldots, j_{m}-1,
$$

and

$$
q_{m}(k)=\frac{\beta_{m}(k+1)}{\beta_{m}(k)}<1, \quad k=j_{m}, \ldots, m-1 .
$$

This implies immediately that as $m \geqslant 8$, finite sequence $(19)$ first strictly increases to the element $\beta_{m}\left(j_{m}\right)$ and then strictly decreases. A mentioned element $\beta_{m}\left(j_{m}\right)$ with $j_{m}$ given by (24) is the sought maximal element of sequence (19). This yields the third statement of Theorem 1. The proof of the theorem is complete.

As some final and at the same time a groundwork for future, let us provide an explicit expression for main characteristics 12. In view of the third statement of Theorem 1, we conclude that

$$
\mu_{2 m}=2^{-2 m} C_{2 m}^{m} \max _{1 \leqslant k \leqslant m} \beta_{m}(k)=2^{-2 m} C_{2 m}^{m} \beta_{m}([(m-1) / 2]), \quad m \geqslant 8 .
$$

Taking into consideration representations $(10)$ and (24), we write a final expression:

$$
\mu_{2 m}=2^{-2 m} C_{2 m}^{m} \frac{1}{2 j_{m}-1} C_{m}^{j_{m}}, \quad j_{m} \equiv\left[\frac{m-1}{2}\right], \quad m \geqslant 8 .
$$

Exactly formula (26) will play a central role in the following proof of Theorem 2.

\section{Proof of Theorem 2}

Here we deal with indices $n=2 m \geqslant 16$ assuming that $m \geqslant 8$. First let us prove asymptotic formula (16). We shall study separately two cases fitting them with standard asymptotics of form (11).

Let $m=2 p$. Then $j_{m} \equiv[(m-1) / 2]=p-1$. According (26), we obtain:

$$
\begin{aligned}
\mu_{4 p} & =2^{-4 p} C_{4 p}^{2 p} \frac{1}{2 p-3} C_{2 p}^{p-1}=\frac{1}{2 p-3} 2^{-4 p} C_{4 p}^{2 p} \frac{(2 p) !}{(p-1) !(p+1) !} \\
& =\frac{p}{(2 p-3)(p+1)} \cdot 2^{-4 p} C_{4 p}^{2 p} \cdot C_{2 p}^{p}, \quad p \in \mathbb{N}, \quad p \geqslant 4 .
\end{aligned}
$$

This is why

$$
\mu_{4 p} \sim \frac{1}{2 p} \cdot \frac{1}{\sqrt{2 \pi p}} \cdot \frac{2^{2 p}}{\sqrt{\pi p}}=\frac{1}{2 \sqrt{2} \pi} \frac{2^{2 p}}{p^{2}}=\frac{\sqrt{2}}{\pi} \frac{2^{2 p}}{(2 p)^{2}}, \quad p \rightarrow \infty,
$$

and this gives formula (16) as $m=2 p$.

Let $m=2 p+1$. Then $j_{m} \equiv[(m-1) / 2]=p$. According (26), we have

$$
\begin{aligned}
\mu_{4 p+2} & =2^{-4 p-2} C_{4 p+2}^{2 p+1} \frac{1}{2 p-1} C_{2 p+1}^{p}=\frac{1}{2 p-1} 2^{-4 p-2} C_{4 p+2}^{2 p+1} \frac{(2 p+1) !}{p !(p+1) !} \\
& =\frac{2 p+1}{(2 p-1)(p+1)} \cdot 2^{-4 p-2} C_{4 p+2}^{2 p+1} \cdot C_{2 p}^{p}, \quad p \in \mathbb{N}, \quad p \geqslant 4 .
\end{aligned}
$$

This is why

$$
\mu_{4 p+2} \sim \frac{1}{p} \cdot \frac{1}{\sqrt{\pi(2 p+1)}} \cdot \frac{2^{2 p}}{\sqrt{\pi p}} \sim \frac{\sqrt{2}}{\pi} \frac{2^{2 p+1}}{(2 p+1)^{2}}, \quad p \rightarrow \infty,
$$

and this gives formula (16) as $m=2 p+1$.

Thus, asymptotics (16) is proven. While proving main non-asymptotic estimate (17), it is again convenient to separate two cases. 
Let $m=2 p$. We define the sequence

$$
\xi_{p}^{(1)}=\frac{\pi}{\sqrt{2}} 2^{-2 p}(2 p)^{2} \mu_{4 p}, \quad p \in \mathbb{N}, \quad p \geqslant 4
$$

Formula $(28)$ ensures that $\xi_{p}^{(1)} \rightarrow 1$ as $p \rightarrow \infty$. Let us show that this sequence tends to one monotonically from below. Employing the last expression in (27), we write explicitly:

$$
\xi_{p}^{(1)}=\frac{\pi}{\sqrt{2}} 2^{-2 p}(2 p)^{2} \cdot \frac{p}{(2 p-3)(p+1)} 2^{-4 p} C_{4 p}^{2 p} C_{2 p}^{p}=\frac{\pi}{\sqrt{2}} 2^{-6 p+2} \frac{p^{3}}{(2 p-3)(p+1)} \frac{(4 p) !}{(2 p) !(p !)^{2}} .
$$

We have the relation:

$$
\begin{aligned}
\frac{\xi_{p+1}^{(1)}}{\xi_{p}^{(1)}} & =\frac{2^{-6 p-4}(p+1)^{3}(4 p+4) !}{(2 p-1)(p+2)(2 p+2) !((p+1) !)^{2}} \cdot \frac{(2 p-3)(p+1)(2 p) !(p !)^{2}}{2^{-6 p+2} p^{3}(4 p) !} \\
& =\frac{(2 p-3)(p+1)^{2}(4 p+1)(4 p+3)}{16 p^{3}(2 p-1)(p+2)}=\frac{32 p^{5}+48 p^{4}-42 p^{3}-109 p^{2}-60 p-9}{32 p^{5}+48 p^{4}-32 p^{3}} .
\end{aligned}
$$

For each $p \geqslant 4$, the numerator of the obtained fraction is less than its denominator. Hence, the above fraction is less than one and sequence (31) strictly decreases to its limit. But then $1<\xi_{p}^{(1)} \leqslant \xi_{4}^{(1)}$ for each $p \geqslant 4$. Here

$$
\xi_{4}^{(1)}=\left.\frac{\pi}{\sqrt{2}} 2^{-6 p+2} \frac{p^{3}}{(2 p-3)(p+1)} \frac{(4 p) !}{(2 p) !(p !)^{2}}\right|_{p=4}=\frac{\pi}{\sqrt{2}} \frac{7 \cdot 9 \cdot 11 \cdot 13}{2^{14}}<1.2215 .
$$

As one can check easily, the scalar gap in the latter estimate is rather small and does not exceed $10^{-5}$. As a result, we arrive at the relation:

$$
1<\xi_{p}^{(1)} \equiv \frac{\pi}{\sqrt{2}} 2^{-2 p}(2 p)^{2} \mu_{4 p}<1.2215, \quad p \in \mathbb{N}, \quad p \geqslant 4 .
$$

Formula (32) implies needed estimate (17) for all even $m=2 p \geqslant 8$.

Assume now that $m=2 p+1$. We proceed in the same way as above. We introduce the sequence

$$
\xi_{p}^{(2)}=\frac{\pi}{\sqrt{2}} 2^{-2 p-1}(2 p+1)^{2} \mu_{4 p+2}, \quad p \in \mathbb{N}, \quad p \geqslant 4 .
$$

Formula 30 ensures that $\xi_{p}^{(2)} \rightarrow 1$ as $p \rightarrow \infty$. Let us show that now this sequence tends to one monotonically from above. Employing the final expression in (29), we write explicitly:

$$
\begin{aligned}
\xi_{p}^{(2)} & =\frac{\pi}{\sqrt{2}} 2^{-2 p-1}(2 p+1)^{2} \cdot \frac{2 p+1}{(2 p-1)(p+1)} 2^{-4 p-2} C_{4 p+2}^{2 p+1} C_{2 p}^{p} \\
& =\frac{\pi}{\sqrt{2}} 2^{-6 p-3} \frac{2 p+1}{(2 p-1)(p+1)} \frac{(4 p+2) !}{(2 p) !(p !)^{2}} .
\end{aligned}
$$

We define the fraction:

$$
\begin{aligned}
\frac{\xi_{p+1}^{(2)}}{\xi_{p}^{(2)}} & =\frac{2^{-6 p-9}(2 p+3)(4 p+6) !}{(2 p+1)(p+2)(2 p+2) !((p+1) !)^{2}} \cdot \frac{(2 p-1)(p+1)(2 p) !(p !)^{2}}{2^{-6 p-3}(2 p+1)(4 p+2) !} \\
& =\frac{128 p^{5}+576 p^{4}+856 p^{3}+348 p^{2}-198 p-135}{128 p^{5}+576 p^{4}+928 p^{3}+688 p^{2}+240 p+32} .
\end{aligned}
$$

For each $p \geqslant 4$, the numerator is less than the denominator. This means that this fraction is less than one and sequence (33) strictly increases to its limit. But then $1<\xi_{p}^{(2)} \leqslant \xi_{4}^{(2)}$ for each $p \geqslant 4$. Here

$$
\xi_{4}^{(2)}=\left.\frac{\pi}{\sqrt{2}} 2^{-6 p-3} \frac{2 p+1}{(2 p-1)(p+1)} \frac{(4 p+2) !}{(2 p) !(p !)^{2}}\right|_{p=4}=\frac{\pi}{\sqrt{2}} \frac{3^{5} \cdot 11 \cdot 13 \cdot 15 \cdot 17}{2^{24}}<1.18 .
$$


As a result, we arrive at the relation:

$$
1<\xi_{p}^{(2)} \equiv \frac{\pi}{\sqrt{2}} 2^{-2 p-1}(2 p+1)^{2} \mu_{4 p+2}<1.18, \quad p \in \mathbb{N}, \quad p \geqslant 4 .
$$

Since $1.18<1.2215$, needed estimate 17 follows 34 for all odd $m=2 p+1 \geqslant 9$. This proves estimate (17) for all $m \geqslant 8$.

We also note that

$$
\frac{\sqrt{2}}{\pi}=0.45015815 \ldots, \quad 1.2215 \cdot \frac{\sqrt{2}}{\pi}=0.54986819 \ldots .
$$

This is why, making (17) a little bit rougher, we obtain close estimate (18). The proof of Theorem 2 is complete.

The meaning of estimate $(17)$ is clear: it confirms that asymptotics $(16)$ expresses rather precise the behavior of quantity $\mu_{2 m}$ for all natural $m \geqslant 8$. By the proof of Theorem 2 one can see easily that the first value $\mu_{16}$ is very close to the upper bound in (17) and as the index $n=2 m$ increases, the numbers $\mu_{2 m}$ go closer to the lower bound coinciding asymptotically as $m \rightarrow \infty$. There are certain nuances in this motion.

While analyzing the proof of Theorem 2, there can arise the idea not to split the proof of estimate (17) into two cases but in view of established asymptotics (16), to introduce one resulting sequence

$$
\gamma_{m}=\frac{\pi}{\sqrt{2}} 2^{-m} m^{2} \mu_{2 m}, \quad m \in \mathbb{N}, \quad m \geqslant 8 .
$$

Then

$$
\gamma_{2 p}=\xi_{p}^{(1)}, \quad \gamma_{2 p+1}=\xi_{p}^{(2)}, \quad p \geqslant 4,
$$

with subsequences $\xi_{p}^{(1)}, \xi_{p}^{(2)}$ in formulae (31), (33), respectively. It is clear that $\gamma_{m} \rightarrow 1$ as $m \rightarrow \infty$. But this tending to one is no longer monotone! Indeed, it is straightforward to check that first all seems to be ok:

$$
\gamma_{12}<\gamma_{11}<\gamma_{10}<\gamma_{9}<\gamma_{8}
$$

but then $\gamma_{13}>\gamma_{12}$, and moreover, $\gamma_{2 p+1}>\gamma_{2 p}$ for all $p \geqslant 6$. It even turns out that $\gamma_{19}>\gamma_{16}$ and so forth. Speaking shortly, although each of two subsequences (36) decreases monotonically, $\gamma_{2 p}=\xi_{p}^{(1)}$ tends to the limit essentially faster than $\gamma_{2 p+1}=\xi_{p}^{(2)}$.

It follows from the said above if we base the proof of estimate (17) on sequence (35), this will require special clarifications. Such details are unlikely to be reasonable. We restrict ourselves by a simple statement: main asymptotic formula (16) contains certain subtleties related to the parity of $m \geqslant 8$.

Nevertheless, formulae (16)- 18 describe well the growth of the maximal coefficient in studied Bernstein polynomials (7). The growth of such rate turns out to be exponential of order $2^{m}=2^{n / 2}$. The neighbouring or equidistant coefficients are of course smaller, but for large indices $n=2 m$ they grow with the same asymptotic rate as in formula (16). Let us rigorously establish this.

\section{BEHAVIOR OF OTHER COEFFICIENTS}

We deal with the coefficients $a_{2 m, 2 k}$ in formula (9) taken for $m \geqslant 8$. We still employ notation (24) letting $j_{m} \equiv[(m-1) / 2]$. We fix a constant value $r \in \mathbb{Z} \backslash\{0\}$, positive or negative.

Similar to the quantity $\mu_{2 m}=\left|a_{2 m, 2 j_{m}}\right|$ corresponding to the maximal (by the absolute value) coefficients $a_{2 m, 2 j_{m}}$, we introduce the characteristics

$$
\mu_{2 m, 2 r}=\left|a_{2 m, 2 j_{m}+2 r}\right|, \quad m \in \mathbb{N}, \quad m \geqslant m_{0}(r),
$$


well-defined for sufficiently large $m$ depending on the choice of $r \in \mathbb{Z} \backslash\{0\}$. Characteristics (37) provides absolute values of the coefficients distant from $a_{2 m, 2 j_{m}}$ exactly by $r$ powers in formal writing of polynomials (6).

Taking into consideration the structure of formula (6), we have the restrictions

$$
1 \leqslant j_{m}+r \leqslant m
$$

By this, we elementary find the values

$$
m \geqslant m_{0}(r)= \begin{cases}\max (2 r-2,8), & r>0, \\ \max (3-2 r, 8), & r<0,\end{cases}
$$

appropriate for defining (37).

In view of the form of coefficients (9) and recalling third statement of Theorem 1, we observe that

$$
\left\{\begin{array}{l}
\mu_{2 m}>\mu_{2 m, 2}>\mu_{2 m, 4}>\ldots, \\
\mu_{2 m}>\mu_{2 m,-2}>\mu_{2 m,-4}>\ldots .
\end{array}\right.
$$

Despite strict inequalities (39), asymptotic behavior of all quantities $\mu_{2 m, 2 r}$ is same as $m \rightarrow \infty$.

Theorem 3. Suppose that for a fixed $r \in \mathbb{Z} \backslash\{0\}$, the quantity $\mu_{2 m, 2 r}$ is defined by formula (37) with restriction (38). Then

$$
\mu_{2 m, 2 r} \sim \frac{\sqrt{2}}{\pi} \frac{2^{m}}{m^{2}}, \quad m \rightarrow \infty,
$$

and this is the same asymptotics as in (16).

Proof. Since result (40) coincides with (16), to obtain the needed relation, it is sufficient to show that $\mu_{2 m, 2 r} / \mu_{2 m} \rightarrow 1$ as $m \rightarrow \infty$. Employing basic formulae (9), (10), we write representations

$$
\begin{gathered}
\mu_{2 m}=\left|a_{2 m, 2 j_{m}}\right|=2^{-2 m} C_{2 m}^{m} \cdot \frac{1}{2 j_{m}-1} C_{m}^{j_{m}}, \\
\mu_{2 m, 2 r}=\left|a_{2 m, 2 j_{m}+2 r}\right|=2^{-2 m} C_{2 m}^{m} \cdot \frac{1}{2 j_{m}+2 r-1} C_{m}^{j_{m}+r} .
\end{gathered}
$$

Hence,

$$
\frac{\mu_{2 m, 2 r}}{\mu_{2 m}}=\frac{2 j_{m}-1}{2 j_{m}+2 r-1} \cdot \frac{C_{m}^{j_{m}+r}}{C_{m}^{j_{m}}}=\frac{2 j_{m}-1}{2 j_{m}+2 r-1} \cdot \frac{j_{m} !\left(m-j_{m}\right) !}{\left(j_{m}+r\right) !\left(m-j_{m}-r\right) !} .
$$

If $m \rightarrow \infty$, then $j_{m} \equiv[(m-1) / 2] \rightarrow \infty$ and $m-j_{m}=m-[(m-1) / 2] \rightarrow \infty$. Therefore,

$$
\frac{2 j_{m}-1}{2 j_{m}+2 r-1} \sim 1, \quad \frac{j_{m} !}{\left(j_{m}+r\right) !} \sim \frac{1}{j_{m}^{r}}, \quad \frac{\left(m-j_{m}\right) !}{\left(m-j_{m}-r\right) !} \sim\left(m-j_{m}\right)^{r}
$$

for each fixed $r \in \mathbb{Z} \backslash\{0\}$. But then

$$
\frac{\mu_{2 m, 2 r}}{\mu_{2 m}} \sim\left(\frac{m-j_{m}}{j_{m}}\right)^{r}, \quad m \rightarrow \infty .
$$

We observe that $j_{m} \equiv[(m-1) / 2]=\left(m-2+\tau_{m}\right) / 2$, where $\tau_{m}=0$ if $m$ is even and $\tau_{m}=1$ if $m$ is odd. This is why

$$
\frac{m-j_{m}}{j_{m}}=\frac{m+2-\tau_{m}}{m-2+\tau_{m}} \rightarrow 1, \quad m \rightarrow \infty .
$$

Combining (41) and (42), we conclude that $\mu_{2 m, 2 r} / \mu_{2 m} \rightarrow 1$ as $m \rightarrow \infty$. As it has been mentioned, this implies needed result (40). The proof is complete. 
Asymptotics (40) means that for sufficiently large indices $n=2 m$, algebraic writing (6) of the studied Bernstein polynomials involves a group of coefficients of approximately same order with huge absolute values. These coefficients are located in the central part of polynomials (6) close to $a_{2 m, 2 j_{m}}$, where $j_{m} \equiv[(m-1) / 2]$. Farther from the center, the character of the behavior of the coefficients changes substantially and at the edges of polynomials (6) completely different laws act.

For instance, the behavior of free coefficients (8) of form $a_{2 m, 0}=2^{-2 m} C_{2 m}^{m}$ is expressed by asymptotics (11), which can be completed by the estimate

$$
\left(1-\frac{1}{8 m}\right) \frac{1}{\sqrt{\pi m}}<2^{-2 m} C_{2 m}^{m}<\left(1-\frac{1}{8 m+1}\right) \frac{1}{\sqrt{\pi m}}, \quad m \in \mathbb{N} .
$$

Two-sided estimate (43) is quite precise and is true for all natural $m$ with no exceptions. One of possible proofs of (43) is given in [17]; for further remarks see [15].

Since

$$
1-\frac{1}{8 m+1}=1-\frac{1}{8 m}+\frac{1}{8 m(8 m+1)}, \quad m \in \mathbb{N},
$$

inequalities (43) imply immediately a known formula

$$
2^{-2 m} C_{2 m}^{m}=\frac{1}{\sqrt{\pi m}}\left(1-\frac{1}{8 m}+O\left(\frac{1}{m^{2}}\right)\right), \quad m \rightarrow \infty,
$$

specifying (11). A finer approximation is of the form

$$
2^{-2 m} C_{2 m}^{m}=\frac{1}{\sqrt{\pi m}}\left(1-\frac{1}{8 m}+\frac{1}{128 m^{2}}+\frac{5}{1024 m^{3}}-\frac{21}{32768 m^{4}}+O\left(\frac{1}{m^{5}}\right)\right) .
$$

For interesting details of such expansions we refer to [18. We note that a universal nonasymptotic estimate (43) should be proved independently and it does not follow asymptotic formulae 44), 45). In any case, the behavior of free coefficients (8) has been found out with a high accuracy.

For the next coefficients $a_{2 m, 2}$ at the powers $x^{2}$, basic formulae (9), (10) give expression:

$$
a_{2 m, 2}=2^{-2 m} C_{2 m}^{m} \cdot m, \quad m \in \mathbb{N} .
$$

Taking into consideration (43), we conclude that

$$
\left(1-\frac{1}{8 m}\right) \sqrt{\frac{m}{\pi}}<a_{2 m, 2}<\left(1-\frac{1}{8 m+1}\right) \sqrt{\frac{m}{\pi}}, \quad m \in \mathbb{N},
$$

with the asymptotics

$$
a_{2 m, 2} \sim \sqrt{\frac{m}{\pi}}, \quad m \rightarrow \infty
$$

Here we observe a slow power growth as $m \rightarrow \infty$.

Finally, according (9), 10), for the higher coefficients in (6) we have the representation:

$$
a_{2 m, 2 m}=2^{-2 m} C_{2 m}^{m} \cdot \frac{(-1)^{m-1}}{2 m-1}, \quad m \in \mathbb{N} .
$$

This is why

$$
\left|a_{2 m, 2 m}\right| \sim \frac{1}{2 \sqrt{\pi} m^{3 / 2}}, \quad m \rightarrow \infty,
$$

that is, the numbers $a_{2 m, 2 m}$ tend to zero by a power law.

In view of explicit expression (7), it is easy to see that the highest coefficient $a_{2 m, 2 m}$ is minimal (by the absolute value) in the polynomial $B_{2 m}(x)$ for each fixed $m \geqslant 2$ (as $m=1$, the polynomial $B_{2}(x)$ contains two same coefficients $\left.a_{2,0}=a_{2,2}=1 / 2\right)$. 
Moreover, as $m \rightarrow \infty$, the coefficients

$$
a_{2 m, 2 m-2}=2^{-2 m} C_{2 m}^{m} \cdot(-1)^{m} \frac{m}{2 m-3}, \quad m \in \mathbb{N}, \quad m \geqslant 2,
$$

tend to zero. All other coefficients, except $a_{2 m, 0}, a_{2 m, 2 m-2}, a_{2 m, 2 m}$, tend to infinity in the sense that

$$
\eta_{2 m} \equiv \min _{1 \leqslant k \leqslant m-2}\left|a_{2 m, 2 k}\right| \rightarrow \infty, \quad m \rightarrow \infty .
$$

Namely, $\eta_{2 m}=\left|a_{2 m, 2 m-4}\right|$ as $m \geqslant 3$ and this implies

$$
\eta_{2 m} \sim \frac{1}{4} \sqrt{\frac{m}{\pi}}, \quad m \rightarrow \infty .
$$

This follows easily explicit expressions (9), 10 in view of main asymptotics (11).

Thus, for sufficiently large indices $B_{2 m}(x)$, almost all coefficients in the studied Bernstein polynomials except three coefficients turn out to be sufficiently large and the central coefficients are not just large but exponentially large (by the absolute value).

Now it is natural to pose a question on the behavior of the entire sum

$$
S_{2 m} \equiv \sum_{k=0}^{m}\left|a_{2 m, 2 k}\right|, \quad m \in \mathbb{N}
$$

This problem is interesting from the point of view of a general theory, see [3].

\section{Asymptotics For the SUM OF ALL COEFFiCIENTS}

Employing representations (8), (9) for the coefficients $a_{2 m, 2 k}$, we write sum (46) as

$$
S_{2 m}=2^{-2 m} C_{2 m}^{m}\left(1+\sum_{k=1}^{m} \frac{1}{2 k-1} C_{m}^{k}\right)=2^{-2 m} C_{2 m}^{m} \sigma_{m},
$$

where

$$
\sigma_{m} \equiv 1+\sum_{k=1}^{m} \frac{1}{2 k-1} C_{m}^{k}, \quad m \in \mathbb{N} .
$$

A character of the technical factor $2^{-2 m} C_{2 m}^{m}$ is clarified by asymptotics (11) and two-sided estimates (43). To study the behavior of quantity $S_{2 m}$, we need to estimate the growth of combinatorial sum (48) as $m \rightarrow \infty$ as accurate as possible. Several first values are

$$
\sigma_{1}=2, \quad \sigma_{2}=\frac{10}{3}, \quad \sigma_{3}=\frac{26}{5}, \quad \sigma_{4}=\frac{278}{35}, \quad \sigma_{5}=\frac{766}{63}, \quad \sigma_{6}=\frac{4366}{231}, \quad \sigma_{7}=\frac{12890}{429} .
$$

A general compact representation for sum (48) seems not to exist. For instance, fundamental handbook [19] provides similar formula (4.2.3.20), but the answer is expressed in terms of some implicit integral. Special computer programs express 48 in terms of the Gauss hypergeometric function. This is why, the following statement is interesting from practical point of view.

Lemma 3. Quantity (48) satisfies the recurrent formula:

$$
\sigma_{m+1}=\frac{2}{2 m+1}\left((m+1) \sigma_{m}+2^{m}-1\right), \quad m \in \mathbb{N} .
$$

Proof. According (48), we have

$$
\begin{aligned}
\sigma_{m+1} & \equiv 1+\sum_{k=1}^{m+1} \frac{1}{2 k-1} C_{m+1}^{k}=1+\sum_{k=1}^{m} \frac{1}{2 k-1}\left(C_{m}^{k}+C_{m}^{k-1}\right)+\frac{1}{2 m+1} \\
& =\sigma_{m}+\sum_{k=0}^{m-1} \frac{1}{2 k+1} C_{m}^{k}+\frac{1}{2 m+1}=\sigma_{m}+\sum_{k=0}^{m} \frac{1}{2 k+1} C_{m}^{k} .
\end{aligned}
$$


We employ the identity

where

$$
\sigma_{m+1}=\sigma_{m}+\pi_{m}, \quad m \in \mathbb{N},
$$

$$
\begin{aligned}
\pi_{m} & \equiv \sum_{k=0}^{m} \frac{1}{2 k+1} C_{m}^{k}=\frac{1}{2 m+1} \sum_{k=0}^{m} \frac{2 k+1+2 m-2 k}{2 k+1} C_{m}^{k} \\
& =\frac{1}{2 m+1}\left(\sum_{k=0}^{m} C_{m}^{k}+2 \sum_{k=0}^{m} \frac{m-k}{2 k+1} C_{m}^{k}\right)=\frac{1}{2 m+1}\left(2^{m}+2 \sum_{k=0}^{m-1} \frac{m-k}{2 k+1} C_{m}^{k}\right) .
\end{aligned}
$$

But $(m-k) C_{m}^{k}=(k+1) C_{m}^{k+1}$ as $k$ ranges from 0 to $m-1$. This is why,

$$
\begin{aligned}
2 \sum_{k=0}^{m-1} \frac{m-k}{2 k+1} C_{m}^{k} & =2 \sum_{k=0}^{m-1} \frac{k+1}{2 k+1} C_{m}^{k+1}=2 \sum_{k=1}^{m} \frac{k}{2 k-1} C_{m}^{k}=\sum_{k=1}^{m} C_{m}^{k}+\sum_{k=1}^{m} \frac{1}{2 k-1} C_{m}^{k} \\
& =2^{m}-1+\sigma_{m}-1=2^{m}+\sigma_{m}-2 .
\end{aligned}
$$

Finally we obtain

$$
\sigma_{m+1}=\sigma_{m}+\pi_{m}=\sigma_{m}+\frac{1}{2 m+1}\left(2^{m}+2^{m}+\sigma_{m}-2\right)=\frac{2}{2 m+1}\left((m+1) \sigma_{m}+2^{m}-1\right) .
$$

The proof is complete.

By means of recurrent formula 49 we establish the following result.

Lemma 4. Quantity (48) satisfies the estimate

$$
\frac{2^{m}}{m-\alpha}<\sigma_{m}<\frac{2^{m}}{m-\beta}, \quad m \in \mathbb{N}, \quad m \geqslant 5,
$$

as $\alpha=2$ and $\beta=3$. The value $\alpha=2$ is asymptotically sharp: for each $\alpha>2$, the lower bound in (50) fails for all indices $m \geqslant m_{0}$ starting from a corresponding $m_{0}=m_{0}(\alpha) \in \mathbb{N}$. On the contrary, the value of $\beta$ can be lessened moving it arbitrarily close to $\alpha=2$ by choosing sufficiently large numbers $m \geqslant m_{1}$ with a corresponding $m_{1}=m_{1}(\beta) \in \mathbb{N}$.

Proof. Let us prove the upper bound. As $\beta=3$, it casts into the form:

$$
\sigma_{m}<\frac{2^{m}}{m-3} \equiv \sigma_{m}^{(+)}, \quad m \in \mathbb{N}, \quad m \geqslant 5
$$

We check the indices $m=5,6,7$ independently. Straightforward calculations give true inequalities:

$$
\begin{aligned}
& \sigma_{5}=\frac{766}{63}=12.1587 \ldots \quad<\sigma_{5}^{(+)}=16, \\
& \sigma_{6}=\frac{4366}{231}=18.9004 \ldots \quad<\sigma_{6}^{(+)}=\frac{64}{3}=21.3333 \ldots, \\
& \sigma_{7}=\frac{12890}{429}=30.0466 \ldots<\sigma_{7}^{(+)}=32 .
\end{aligned}
$$

In what follows we argue by induction.

Assume that estimate (51) is true for some $m \geqslant 7$. Then according (49), we have

$$
\begin{aligned}
\sigma_{m+1} & =\frac{2}{2 m+1}\left((m+1) \sigma_{m}+2^{m}-1\right)<\frac{2}{2 m+1}\left((m+1) \sigma_{m}+2^{m}\right) \\
& <\frac{2}{2 m+1}\left((m+1) \frac{2^{m}}{m-3}+2^{m}\right)=\frac{2^{m+1}}{2 m+1}\left(\frac{m+1}{m-3}+1\right) \\
& =\frac{2^{m+1}(2 m-2)}{(2 m+1)(m-3)} \leqslant \frac{2^{m+1}}{m-2} \equiv \sigma_{m+1}^{(+)}
\end{aligned}
$$


since

$$
\frac{1}{m-2}-\frac{2 m-2}{(2 m+1)(m-3)}=\frac{m-7}{(m-2)(2 m+1)(m-3)} \geqslant 0
$$

as $m \geqslant 7$. Thus, estimate (51) is true for all $m \geqslant 5$.

Strictly saying, estimate (51) is true also for $m=4$, but this case is not included in general formula (50) since as $m=4$ the corresponding lower bound is not guaranteed.

Let us prove the lower bound. As $\alpha=2$, it becomes

$$
\sigma_{m}>\frac{2^{m}}{m-2} \equiv \sigma_{m}^{(-)}, \quad m \in \mathbb{N}, \quad m \geqslant 5
$$

Since

$$
\sigma_{5}=\frac{766}{63}=12.1587 \ldots>\sigma_{5}^{(-)}=\frac{32}{3}=10.6666 \ldots,
$$

for $m=5$ estimate $(52)$ is satisfied. Further we argue by induction. Assume that estimate 52 is true for some $m \geqslant 5$. According (49), we have

$$
\begin{aligned}
\sigma_{m+1} & =\frac{2}{2 m+1}\left((m+1) \sigma_{m}+2^{m}-1\right)>\frac{2}{2 m+1}\left((m+1) \frac{2^{m}}{m-2}+2^{m}\right)-\frac{2}{2 m+1} \\
& =\frac{2^{m+1}}{2 m+1}\left(\frac{m+1}{m-2}+1\right)-\frac{2}{2 m+1}=\frac{2^{m+1}(2 m-1)}{(2 m+1)(m-2)}-\frac{2}{2 m+1}>\frac{2^{m+1}}{m-1} \equiv \sigma_{m+1}^{(-)}
\end{aligned}
$$

since

$$
\frac{2^{m+1}(2 m-1)}{(2 m+1)(m-2)}-\frac{2^{m+1}}{m-1}=\frac{3 \cdot 2^{m+1}}{(2 m+1)(m-2)(m-1)}>\frac{2}{2 m+1}
$$

thanks to the obvious relation $3 \cdot 2^{m}>(m-2)(m-1)$ being true for all $m \in \mathbb{N}$. Thus, assertion (52) is established and estimate (50) with $\alpha=2$ and $\beta=3$ is completely proven.

Let us study the accuracy of the values $\alpha=2$ and $\beta=3$ in estimate (50). Employing the elementary transformation

$$
\frac{1}{m-\gamma}=\frac{1}{m}+\frac{\gamma m}{m-\gamma} \frac{1}{m^{2}}, \quad m>\gamma>0
$$

we write 50 in the following equivalent form

$$
\frac{2^{m}}{m}+\frac{\alpha m}{m-\alpha} \frac{2^{m}}{m^{2}}<\sigma_{m}<\frac{2^{m}}{m}+\frac{\beta m}{m-\beta} \frac{2^{m}}{m^{2}}, \quad m \in \mathbb{N}, \quad m \geqslant 5,
$$

with values $\alpha=2$ and $\beta=3$. We denote temporarily

$$
x_{m}=\sigma_{m}-\frac{2^{m}}{m}, \quad y_{m}=\frac{2^{m}}{m^{2}}, \quad m \in \mathbb{N} .
$$

Then (53) is reduced to the form

$$
\frac{\alpha m}{m-\alpha}<\frac{x_{m}}{y_{m}}<\frac{\beta m}{m-\beta}, \quad m \in \mathbb{N}, \quad m \geqslant 5,
$$

where $\alpha=2, \beta=3$. It is clear that

$$
\lim _{m \rightarrow \infty} \frac{\alpha m}{m-\alpha}=\alpha, \quad \lim _{m \rightarrow \infty} \frac{\beta m}{m-\beta}=\beta .
$$

Let us show the existence of the limit

$$
\lim _{m \rightarrow \infty} \frac{x_{m}}{y_{m}}=2 .
$$

This implies immediately that the value $\alpha=2$ in formula (55) is asymptotically sharp, while the value $\beta=3$ can be lessen in the interval $2<\beta \leqslant 3$ moving $\beta$ arbitrarily close to $\alpha=2$ by choosing sufficiently large indices $m \geqslant m_{1}=m_{1}(\beta)$. 
We prove existence of limit (56) for quotient of sequence (54) by applying usual Stolz-Cesàro theorem [20]. In view of definition (54) and recurrent formula (49) we have

$$
\begin{aligned}
x_{m+1}-x_{m} & =\sigma_{m+1}-\frac{2^{m+1}}{m+1}-\sigma_{m}+\frac{2^{m}}{m}=\frac{2 m+2}{2 m+1} \cdot \sigma_{m}+\frac{2^{m+1}-2}{2 m+1}-\frac{2^{m+1}}{m+1}-\sigma_{m}+\frac{2^{m}}{m} \\
& =\frac{1}{2 m+1} \cdot \sigma_{m}+\frac{3 m+1}{m(m+1)(2 m+1)} \cdot 2^{m}-\frac{2}{2 m+1} .
\end{aligned}
$$

At that,

$$
y_{m+1}-y_{m}=\frac{2^{m+1}}{(m+1)^{2}}-\frac{2^{m}}{m^{2}}=\frac{m^{2}-2 m-1}{m^{2}(m+1)^{2}} \cdot 2^{m} .
$$

Sequence $y_{m}$ strictly increases to $+\infty$ as $m \geqslant 3$. The assumptions of the Stolz-Cesàro theorem are satisfied. For the quotient of the difference we have

$$
\frac{x_{m+1}-x_{m}}{y_{m+1}-y_{m}}=\frac{m(m+1)}{m^{2}-2 m-1}\left(\frac{m(m+1)}{2 m+1} \cdot 2^{-m} \sigma_{m}+\frac{3 m+1}{2 m+1}-\frac{2 m(m+1)}{2 m+1} \cdot 2^{-m}\right) .
$$

We note that $m \cdot 2^{-m} \sigma_{m} \rightarrow 1$ as $m \rightarrow \infty$ according proven estimate (50). This is why

$$
\lim _{m \rightarrow \infty} \frac{x_{m+1}-x_{m}}{y_{m+1}-y_{m}}=1 \cdot\left(\frac{1}{2} \cdot 1+\frac{3}{2}-0\right)=2 .
$$

Hence, by the Stolz-Cesàro theorem, we obtain limit (56). Comparing (56) with inequality (55), we establish all needed statements on asymptotic sharpness of the value $\alpha=2$ and on lessening the value $\beta=3$. These statements are extended immediately to equivalent formula (50). The proof is complete.

The obtained result imply the following corollary.

Lemma 5. Quantity 48 satisfies the asymptotics

$$
\sigma_{m} \sim \frac{2^{m}}{m}, \quad m \rightarrow \infty
$$

more precisely,

$$
\sigma_{m}=\frac{2^{m}}{m}\left(1+\frac{2}{m}+o\left(\frac{1}{m}\right)\right), \quad m \rightarrow \infty .
$$

Proof. Asymptotics (57) follows estimate (50). Specified formula (58) is equivalent to found limit (56) in view of notations introduced in (54). The proof is complete.

Now we are in position to find out the behavior of sum $(46)$ formed by the coefficients of the studied Bernstein polynomials. For the sake of convenience, we give several first values:

$$
S_{2}=1, \quad S_{4}=\frac{5}{4}, \quad S_{6}=\frac{13}{8}, \quad S_{8}=\frac{139}{64}, \quad S_{10}=\frac{383}{128}, \quad S_{12}=\frac{2183}{512}, \quad S_{14}=\frac{6445}{1024} .
$$

As the index $n=2 m$ increases, the growth of the sequence $S_{2 m}$ becomes more visible, but a real growth rate is clarified just by the following statement.

Theorem 4. Suppose that the sums $S_{2 m}$ are defined by formula (46) with coefficients (8), (9) of Bernstein polynomials (7). Then the sequence $S_{2 m}$ satisfies the asymptotics:

$$
S_{2 m} \sim \frac{1}{\sqrt{\pi}} \frac{2^{m}}{m^{3 / 2}}, \quad m \rightarrow \infty
$$

more precisely,

$$
S_{2 m}=\frac{1}{\sqrt{\pi}} \frac{2^{m}}{m^{3 / 2}}\left(1+\frac{15}{8 m}+o\left(\frac{1}{m}\right)\right), \quad m \rightarrow \infty
$$


Moreover, the estimate holds:

$$
\left(1-\frac{1}{8 m}\right) \frac{2^{m}}{\sqrt{\pi m}(m-2)}<S_{2 m}<\left(1-\frac{1}{8 m+1}\right) \frac{2^{m}}{\sqrt{\pi m}(m-3)}
$$

valid for all natural $m \geqslant 5$.

Proof. Thanks to (47), let us employ the representation $S_{2 m}=2^{-2 m} C_{2 m}^{m} \sigma_{m}$ with the factors $\sigma_{m}$ in formula (48). Taking into consideration asymptotics (11) and (57), we obtain needed relation (59). More gentle formula (60) is found by multiplying (44) and (58). In the same way, taking into consideration previous estimates (43) and (50) (the latter is used with the values $\alpha=2$ and $\beta=3$ ), we establish two-sided estimate (61) for all natural $m \geqslant 5$. We also note that as $m=4$, estimate $(61)$ fails since for the lower bound we obtain

$$
\left.\left(1-\frac{1}{8 m}\right) \frac{2^{m}}{\sqrt{\pi m}(m-2)}\right|_{m=4}=\frac{31}{8 \sqrt{\pi}}=2.186234 \ldots>S_{8}=\frac{139}{64}=2.171875,
$$

which contradicts (61). The proof is complete.

It is interesting to compare the established facts with one general result by J.A. Roulier. In his work [3], there was obtained an universal estimate for the sum of the coefficients of the Bernstein polynomials on a segment $[a, b]$ with the condition $a \leqslant 0<1 \leqslant b$. For the symmetric segment $[-1,1]$, the Roulier result gives the following.

Let $f \in C[-1,1]$ be a function with the Bernstein polynomials $B_{n}(f, x)$ written by formula (1). We write in the algebraic form:

$$
B_{n}(f, x)=\sum_{k=0}^{n} a_{n, k}(f) x^{k}, \quad n \in \mathbb{N}
$$

and we denote

$$
S_{n}(f) \equiv \sum_{k=0}^{n}\left|a_{n, k}(f)\right|, \quad n \in \mathbb{N} .
$$

Then, according Theorem 1 in [3], the estimate holds:

$$
S_{n}(f) \leqslant 2^{n}\|f\|, \quad n \in \mathbb{N},
$$

where $\|\cdot\|$ is the usual supremum norm on $[-1,1]$.

We observe that for the function $f(x)=|x|$ on $[-1,1]$, estimate (64) turns out to be rather rough. Indeed, in this case, taking into consideration gluing rule (4) and passing from general formulae (62), (63) to particular ones (6), (46), we obtain:

$$
S_{2 m+1}(f)=S_{2 m}(f)=S_{2 m}, \quad m \in \mathbb{N} .
$$

Therefore, as $n=2 m$, by formula $(59)$ we have

$$
S_{n}(f)=S_{2 m} \sim \frac{1}{\sqrt{\pi}} \frac{2^{m}}{m^{3 / 2}}=\frac{2 \sqrt{2}}{\sqrt{\pi}} \frac{2^{n / 2}}{n^{3 / 2}}, \quad n=2 m \rightarrow \infty,
$$

and as $n=2 m+1$, we get

$$
S_{n}(f)=S_{2 m} \sim \frac{1}{\sqrt{\pi}} \frac{2^{m}}{m^{3 / 2}} \sim \frac{2}{\sqrt{\pi}} \frac{2^{n / 2}}{n^{3 / 2}}, \quad n=2 m+1 \rightarrow \infty .
$$

The asymptotics for even and odd indices is formally different but in any case

$$
S_{n}(f)=o\left(2^{n / 2}\right), \quad n \rightarrow \infty,
$$

with the exponent function $2^{n / 2}$ and not $2^{n}$ as in (64). The comparison is rather clear. 
In view of the said above, a natural problem arises: by choosing a break point $p / q \in(-1,1)$, find an example of a simple rational modulus

$$
f(x)=|q x-p|, \quad x \in[-1,1],
$$

for which the coefficients in Bernstein polynomials (1) in writing (62) have the maximal growth as $n \rightarrow \infty$. Whether there exists such example with substantial exceeding of growth (65) is still unclear. The situations likely differs from that on the standard segment [0,1], cf. [9].

It could be quite interesting to show the sharpness of Roulier estimate $(64)$ on $[-1,1]$ or to obtain another universal estimate for quantity (63) applicable for all functions $f \in C[-1,1]$, strengthening result (64) and being extremely sharp on the class $C[-1,1]$.

Remark on proofreading: recently there was a substantial advantage in this problem, see [23].

\section{ACKNOWLEDGEMENTS}

The authors thank participants of a series of conference for profound discussions of the results partially reported in talks [21], [22]. We express a special gratitude to A.Yu. Trynin, who pointed out work [1] that allowed us to related our study with general direction [1]-[7]. We also mention a friendly help by D.G. Tsvetkovich, who checked carefully all out calculations.

\section{BIBLIOGRAPHY}

1. J.D. Stafney. A permissible restriction on the coefficients in uniform polynomial approximation to $C[0,1]$ // Duke Math. J. 34:3, 393-396 (1967).

2. S.Ya. Khavinson. Permissible values of coefficients of polynomials in uniform approximation of continuous functions // Matem. Zamet. 6:5, 619-625 (1969). [Math. Notes. 6:5, 834-838 (1969).]

3. J.A. Roulier. Permissible bounds on the coefficients of approximating polynomials // J. Approx. Theory. 3:2, 117-122 (1970).

4. J.A. Roulier. Restrictions on the coefficients of approximating polynomials // J. Approx. Theory. 6:3, 276-282 (1972).

5. V.I. Gurarii, M.A. Meletidi. On estimates of the coefficients of polynomials approximating continuous functions // Funkts. Anal. Pril. 5:1, 73-75 (1971). [Funkt. Anal. Appl. 5:1, 60-62 (1971).]

6. M. Golitschek, D. Leviatan. Permissible bounds on the coefficients of approximating polynomials with real or complex exponents // J. Math. Anal. Appl. 60:1, 123-138 (1977).

7. O.A. Muradyan, S.Ya. Khavinson. Absolute values of the coefficients of the polynomials in Weierstrass's approximation theorem // Matem. Zamet. 22:2, 269-276 (1977). [Math. Notes. 22:2, 641645 (1977).]

8. I.V. Tikhonov, V.B. Sherstyukov. The module function approximation by Bernstein polynomials // Vestn. Chelyabinsk. Univ. Matem. Mekh. Inform. 15:26, 6-40 (2012). (in Russian).

9. I.V. Tikhonov, V.B. Sherstyukov. On behaivor of coefficients of Bernstein polynomials in algerbraic writing on a standard segment // in "Certain topical problems of modern mathematics and mathematical education". Proc. Sci. Conf. "Herzen Readings - 2015". Herzen Univ., St.-Petersburg, 115-121 (2015). (in Russian).

10. I.V. Tikhonov, V.B. Sherstyukov, M.A. Petrosova. Gluing rule for Bernstein polynomials on the symmetric interval // Izv. Saratov. Univ. Nov. Ser. Matem. Mekh. Inform. 15:3, 288-300 (2015). (in Russian).

11. I.V. Tikhonov, V.B. Sherstyukov, M.A. Petrosova. Bernstein polynomials for a standard module function on the symmetric interval // Izv. Saratov. Univ. Nov. Ser. Matem. Mekh. Inform. 16:4, 425-435 (2016). (in Russian).

12. I.P. Natanson. Constructive function theory. GITTL, Moscow (1949). [Vol. I-III, Frederick Ungar Publishing Co., New York (1964-1965).]

13. G.G. Lorentz. Bernstein Polynomials. University of Toronto Press, Toronto (1953). 
14. V.S. Vidensky. Bernstein polynomials. Tutorial for a special course. Leningrad State Pedagog. Inst. named after A.I. Herzen, Leningrad (1990). (in Russian).

15. I.V. Tikhonov, V.B. Sherstyukov, M.A. Petrosova. Bernstein polynomials: old and new // in "Mathematical Forum". Vol. 8. Part 1. Studies in Mathematical Analysis. South Math. Inst. VSC RAS, 126-175 (2014). (in Russian).

16. J. Bustamante. Bernstein operators and their properties. Birkhäuser, Basel (2017).

17. A.Yu. Popov. Two-sided estimates of sum of values of a function at integer points and their applications. Pereslavl Univ., Pereslavl-Zalesskii (2016). (in Russian).

18. D.E. Knuth, I. Vardi. The asymptotic expansion of the middle binomial coefficient // Amer. Math. Monthly. 97:7, 629-630 (1990).

19. A.P. Prudnikov, Yu.A. Brychkov, O.I. Marichev. Integrals and series. Elementary functions. Nauka, Moscow (1981). [Integrals and series. Vol. 1. Elementary functions. Gordon \& Breach Science Publ. New York (1986).]

20. V.A. Il'in, E.G. Poznyak. Foundations of mathematical analysis. Nauka, Moscow (1965). (in Russian).

21. M.A. Petrosova. On growth rate of maximal coefficients in Bernstein polynomials taken of a symmetric modulus function on a symmetric segment // in "Modern problems of theory of functions and applications". Proc. of 18th Intern. Saratov Winter School. "Nauchnaya Kniga" Publ., Ltd., Saratov, 209-211 (2016). (in Russian).

22. M.A. Petrosova. On behavior of coefficients in Bernstein polynomials taken of a symmetric modulus function on a symmetric segment // in "Mathematics and Informatics". Proc. of Intern. Conf. Moscow State Pedagog. Univ., Moscow, 77-79 (2016). (in Russian).

23. I.V. Tikhonov, V.B. Sherstyukov, M.A. Petrosova. New studies related to algebraic writing of Bernstein polynomials on a symmetric segment // Systems Comp. Math. Appl. 19, 336-347 (2018). (in Russian).

Margarita Arsenovna Petrosova, Moscow State Pedagogical University,

Krasnoprudnaya str. 14,

107140, Moscow, Russia

E-mail: petrosova05@mail.ru

Ivan Vladimirovich Tikhonov,

Lomonosov Moscow State University,

Leninskie gory 1-52, GSP-1,

119991, Moscow, Russia

E-mail: ivtikh@mail.ru

Vladimir Borisovich Sherstyukov,

National Research Nuclear University "MEPhI",

Kashirskoe road 31,

115409, Moscow, Russia

E-mail: shervb73@gmail.com 